\title{
Post-traumatic amnesia: still a valuable yardstick
}

\author{
J T L Wilson, G M Teasdale, D M Hadley, K D Wiedmann, D Lang
}

\begin{abstract}
Records of coma and post-traumatic amnesia (PTA) were collected for a group of 38 patients with closed head injury. The results confirmed earlier studies indicating that patients may have short or negligible coma but report prolonged PTA. Comparison of eight patients with prolonged PTA ( $>7$ days) and short coma ( $<6$ hours) with the rest of the group on MRI in the acute stage showed that these patients had significantly more extensive hemispheric damage. In the group as a whole both coma and PTA were related to the number of areas in central brain structures in which lesions were detected, but only PTA was significantly related to the number of hemispheric areas in which lesions were found. It is concluded that although both coma and PTA are related to brain damage they reflect disparate patterns of lesions. Assessment of PTA can thus provide additional information concerning severity of injury.
\end{abstract}

$(\Im$ Neurol Neurosurg Psychiatry 1994;57:198-201)

The most widely accepted methods of assessing the severity of diffuse brain damage after trauma are based on observation of disturbance of consciousness. For many years the duration of post-traumatic amnesia (PTA), obtained retrospectively at interview, was the main method used to assess the extent of brain damage after head injury. ${ }^{12}$ An editorial in the Lancet concluded that "PTA is the best yardstick for assessing severity of head injury". ${ }^{3}$ The introduction of the Glasgow Coma Scale (GCS) allowed precise definition of conscious level, and facilitated consistent measurement of depth and duration of coma. ${ }^{4}$ Assessment of the GCS is based on independent objective observation at the time of injury rather than on the patient's report weeks, months or years later, and is preferred in many centres as a measure of injury severity.

There is an assumption that coma depth and duration and post-traumatic amnesia are closely related, that they may reflect different aspects of a common mechanism, and that they can be exchanged for one another. However, when PTA and coma have been compared the relationship between them has proven to be only moderately close. ${ }^{56}$ Furthermore, studies investigating PTA and coma as predictors of outcome show sometimes that coma relates to outcome while PTA does not, ${ }^{7}$ or vice versa. ${ }^{8}$ Such discrepancies have usually been attributed to problems in accurately measuring PTA. ${ }^{10}$ Another possibility is that coma and PTA reflect different aspects of brain damage and that they are thus not equivalent.

The purpose of the present investigation was to examine the relationship between duration of coma and PTA, and to determine how these measures of severity of injury related to brain damage visualised by MRI.

\section{Method}

Forty eight patients with traumatic head injury admitted to a regional neurosurgical unit were initially entered in the study. Patients were excluded from the present analysis if they had delayed coma due to haematoma, or if relevant imaging or follow up data were not available. There were 38 patients (30 male) for whom a full set of data was present. The age range was 16 to 73 years (mean 35 years). Road traffic accidents (RTAs) accounted for 17 injuries, falls 19 injuries, and assault 2 injuries. Patient records were examined to determine the GCS on admission and duration of coma. The patient was considered to be out of coma if their eyes were open, they spoke comprehensible words, or they obeyed commands. Coma duration was taken to be the interval between injury and the point at which the patient was consistently out of coma by these criteria. Patients were followed up 5 to 12 months after injury and duration of PTA was determined at interview. The duration of PTA was taken to be the interval between injury and the time at which the patient regained continuous memory. Hospital records were consulted to establish landmarks in the acute stage from which the duration of PTA could be gauged. For most patients a close relative was also interviewed concerning the chronology of events and recovery of memory. A series of neuropsychological measures was also administered at follow up. ${ }^{11}$

MRI was performed within the first week after injury using a Picker Vista 1100, 0.15 Tesla resistive system. Details of imaging methods are given elsewhere. ${ }^{12}$ The presence of lesions on acute MRI was coded for 21 different brain regions for these patients. There were 12 hemispheric regions, comprising left and right areas for each of the following: orbito frontal, frontal, temporal pole, 
temporal, parietal and occipital. A further nine areas in central brain structures were coded: right and left basal ganglia; genu, trunk, and splenium of the corpus callosum; left and right brainstem; left and right cerebellum. For the purpose of the present analysis three overall indices of brain damage were derived for each patient: (1) total number of areas in which lesions were detected; (2) number of hemispheric areas in which lesions were detected; (3) number of areas in central structures in which lesions were detected.

\section{Results}

Coma duration ranged from nil to 3 weeks, and duration of PTA from nil to 3 months. The distributions of coma duration and PTA duration are compared in table 1 . The Spearman rank order correlation between initial GCS and duration of coma was -0.83 , $\mathrm{p}<0.001$, indicating a relatively strong relationship between these measures; however, the correlation between duration of coma and duration of PTA, 0.50, p $<0.001$, was indicative of only a moderate agreement. The table suggests that for patients with coma lasting six hours or more there is concordance with the PTA estimate of the severity of injury: the results indicate that coma of this duration corresponds to a PTA of over 7 days. For patients in coma for less than six hours there is much less agreement with PTA. The main discrepancy in the measures of injury severity arises from cases with prolonged PTA but without prolonged coma.

Eight patients were in coma for less than six hours but had a PTA longer than 1 week (table 1); three of these patients had been only briefly unconscious, if at all. The mean age of these eight cases, 44 years, was not significantly different from the rest of the patients, mean age $33, t=1 \cdot 79$, ns. Two of the eight patients had claims for compensation pending; in the rest of the group 15 patients of 30 had compensation claims

Table 1 Distribution of PTA and coma duration

\begin{tabular}{lllll}
\hline & \multicolumn{3}{l}{ PTA } & \\
\cline { 3 - 5 } & & $\begin{array}{l}1 \text { day } \\
\text { or less }\end{array}$ & $2-7$ days & $\begin{array}{l}\text { more than } \\
7 \text { days }\end{array}$ \\
\hline \multirow{2}{*}{ Coma } & Less than 6 hours & 7 & 9 & 8 \\
\cline { 2 - 5 } 6 hours or more & 0 & 2 & 12 \\
\hline
\end{tabular}

Table 2 Spearman correlations between PTA and coma and indices of the extent of brain damage derived from MRI

\begin{tabular}{llll}
\hline \multicolumn{4}{c}{$\begin{array}{l}\text { Number of areas with } \\
\text { lesions detected by } M R\end{array}$} \\
\cline { 2 - 4 } & $\begin{array}{l}\text { Hemispheric } \\
\text { areas }\end{array}$ & $\begin{array}{l}\text { Central } \\
\text { areas }\end{array}$ & $\begin{array}{l}\text { Total } \\
\text { areas }\end{array}$ \\
\hline Arrival GCS & -0.12 & $-0.42^{\star \star}$ & $-0.29^{\star}$ \\
Coma duration & 0.01 & $0.45^{\star \star}$ & 0.21 \\
PTA duration & $0.37^{\star}$ & $0.57^{\star \star \star}$ & $0.57^{\star \star \star}$ \\
PTA-Coma & $0.37^{\star}$ & $0.56^{\star \star \star}$ & $0.56^{\star \star \star}$ \\
\hline Notes. ${ }^{\star}=\mathrm{p}<0.05,{ }^{\star \star}=\mathrm{p}<0.01,{ }^{\star \star \star}=\mathrm{p}<0.001$.
\end{tabular}

which had not been settled. In seven of the eight patients the injuries had been caused by falls. Comparison of the results of acute MRI of the eight patients with prolonged PTA with the rest of the group showed that there was not a significant difference in the mean number of areas in which lesions were detected, 7.5 and $5 \cdot 1$, respectively, $t=1.98$, ns. However, in the patients with prolonged PTA the mean number of hemispheric areas with a lesion present, 5.9, was significantly greater than in the rest of the patients, mean $=3.7, \mathrm{t}=2.2, \mathrm{p}<0.05$ (two-tailed). Thus patients with short coma but long PTA had suffered significantly greater brain damage, particularly in hemispheric areas. Examination of the distribution of lesions in particular brain areas did not reveal any patterns of injury which were specific to patients with prolonged PTA.

To establish if there was a link between PTA and brain damage in patients with short coma a comparison was made between the eight patients with prolonged PTA and the seven patients who had short PTA. These two groups of patients were matched closely in age (mean 44 years in both groups). Patients with short PTA had a mean of 3.0 hemispheric area with lesions present, compared with 5.9 in those with long PTA. The groups were compared on a set of 11 neuropsychological tests performed at follow up. The two groups were not significantly different on a measure of premorbid IQ (National Adult Reading Test). They did not differ significantly on Verbal subtests of the Wechsler Adult Intelligence Scale (Similarities, Vocabulary, Digit Span), but the group with prolonged PTA performed significantly more poorly on Performance subtests (Digit Symbol, $t=3.25, p<0.01$; Block Design, $\mathrm{t}=2.24, \quad \mathrm{p}<0.05 ;$ Object Assembly, $\mathrm{t}=$ $2 \cdot 47, \mathrm{p}<0.05)$. There was no difference between groups on two measures of verbal memory (Logical Memory and Associate Learning), however, patients with prolonged PTA performed more poorly on a test of visual memory (Rey Figure Recall, $t=3 \cdot 66$, $\mathrm{p}<0.01$ ). Patients with prolonged PTA were also significantly less proficient on a measure of word fluency $(t=2.98, p<0.05)$. These results are in line with previous reports that longer PTA is related to poor cognitive performance, and provide supporting evidence that the patients with prolonged PTA in the current study had suffered significant brain damage.

The data were analysed to investigate the relationships between coma, PTA and lesions detected by MRI in the group as a whole. Rank order correlations showed that both hemispheric lesions and central lesions were significantly related to total lesions, rho $=$ $0.90, \mathrm{p}<0.001$ and rho $=0.60, \mathrm{p}<0.001$, respectively, but not to each other, rho $=$ $0 \cdot 21$, ns. Correlations between these MRI derived indices of severity of brain damage and coma and PTA duration are given in table 2. As can be seen both PTA and coma are related to lesions in central structures, but 
only PTA is related to hemispheric lesions. The relationship between PTA and total number of areas damaged is stronger than for either arrival GCS or coma duration. PTA duration can be calculated either from the time of injury, ${ }^{1}$ or from the end of coma. ${ }^{13}$ Table 2 shows that the two methods of computing PTA give very similar results.

\section{Discussion}

The study confirmed that duration of PTA and coma is only modestly correlated, and that there is a subgroup of patients with short or negligible coma and prolonged PTA. ${ }^{5614}$ Patients with prolonged PTA showed more extensive brain damage on MRI compared with the rest of the group. These patients also showed more neuropsychological impairment in comparison to a group with short PTA and short coma. The study thus indicates that patients reporting prolonged PTA have suffered significant brain damage which is not adequately reflected by assessing either the depth or duration of coma alone.

There are some evident limitations to assessing PTA by interview. A potential problem is the effect of secondary systemic disorders such as hypoxia which may lead to a loss of memory, in addition to the effects of the initial injury. Review of the details of the case notes may reveal whether this is likely to be the reason for the reported amnesia. As far as we could ascertain this did not apply to any of the cases in our study who reported prolonged PTA. It must also be noted that the reliability of assessment of PTA remains unknown. There may be variation in estimates obtained on different occasions, or by different interviewers. The subjective nature of this form of PTA assessment will continue to be an important limitation. Exaggeration for monetary benefit is also sometimes offered as an explanation for the reports of an unduly long PTA. However, patients with prolonged PTA and short coma were less likely to be involved in a claim for compensation than other patients in the present study. A further common pitfall in measuring PTA is mistaking islands of memory for the end of amnesia..$^{10}$ However, islands would lead to PTA duration being underestimated, not unexpectedly prolonged as we found.

The results also confirm previous findings in indicating that prolonged PTA cannot be attributed to the age of the patients. ${ }^{6}$ Nonetheless, patients with prolonged PTA were on average ten years older than the group as a whole. It seems probable that this reflects differences in the aetiologies of the injuries, and specifically the fact that most of the injuries in patients with prolonged PTA and short coma had been caused by falls. In the group as a whole there was an age difference between patients with injuries caused by falls, mean age $=41$ years, and RTAs, mean age $=30$ years. Similar age differences have been noted by others previously. ${ }^{15}$ The present findings indicate that falls which do not produce prolonged coma can nonetheless result in extensive injuries to the cerebral hemispheres.

The findings support the idea that coma duration and PTA reflect different aspects of brain damage. The results show that GCS on arrival at hospital and the duration of coma relate to the number of areas in central brain structures with detectable lesions. This finding is consistent with earlier work showing that depth of lesions detected by MRI was related to level of consciousness on admission. ${ }^{16}$ It also agrees with the view that disturbances of consciousness are specifically related to disruption of the functions of subcortical activating mechanisms. ${ }^{17}$ On the other hand, PTA was related not only to lesions in central structures but also to lesions in hemispheric areas. Persistent amnesia is associated with damage to specific groups of structures: the medial temporal lobes, basal forebrain, and midline diencephalon. ${ }^{18}$ Thus a relationship between amnesia and overall cerebral damage seems paradoxical. A common view is that trauma necessarily involves diffuse damage to white matter, and the more severe the injury the greater the extent of this damage. To explain the relationship with PTA it must be assumed that the structures involved in episodic memory are particularly vulnerable to the effects of such damage, either through disconnection from other brain regions, or from disruption of their intrinsic circuitry. An alternative possibility, not invoking diffuse white matter damage, is that PTA may be related to lesions in specific areas, such as subfrontal areas or temporal poles, which themselves show a strong relationship with overall brain damage in head injury. ${ }^{11}$ The reason for the relationship between duration of PTA and overall brain damage remains unknown.

The correlations between the conventional measures of severity and MRI indices of brain damage are themselves only moderate. There are probably a number of reasons for the lack of a strong agreement. This may reflect problems in precisely measuring the duration of either coma or PTA. It may also reflect inadequacies in the MRI index used: size of lesions might, for example, be a better index, and perhaps lesions in different positions should be weighted differently in the calculation. Furthermore, MRI does not detect all lesions in head injury, and, in particular, it does not resolve the microscopic damage characteristic of diffuse axonal injury.

It is clear that acute MRI cannot replace the use of GCS and PTA in the practical assessment of severity of brain injury after trauma. However, people using these measures should be aware of the evidence from MRI that they reflect different patterns of underlying brain damage. On the present results PTA yields a less specific and more global measure of brain damage than coma depth or duration. GCS will continue to be preferred as an objective measure of initial severity of injury. However, careful assessment of PTA yields additional information. In particular a finding of prolonged PTA in 
the absence of prolonged coma should not simply be dismissed as fabrication. or error, rather it should serve to signal the possibility of significant hemispheric damage.

Data collection was funded by a grant from the Medical Data collection was funded by a grant from the Medica
Research Council. This work is currently supported by the Research Council.
Wellcome Trust.

1 Russell W R. Cerebral involvement in head injury. Brain 1932;55:549-603.

2 Symonds C P, Russell W R. Accidental head injuries. Lancet 1943; i:7-9.

3 The best yardstick we have. Editorial Lancet 1961;ii: 1445-6.

4 Teasdale G, Jennett B. Assessment of coma and impaired consciousness: A practical scale. Lancet 1974;ii:81-84.

5 Levin H S, Papanicolaou A, Eisenberg H M. Observations on amnesia after nonmissile head injury. In: Squire $L$, Butters N, eds. Neuropsychology of memory. London: Guilford Press: 1984.

6 Teasdale G, Brooks D N. Traumatic amnesia. In: Fredriks I A M, ed. Handbook of clinical neurology, vol 1(45): Clinical neuropsychology. Amsterdam: Elsevier, 1984:185-191.

7 Alexandre A, Colombo F, Nertempi P, Benedetti A. Cognitive outcome and early indices of severity of injury 7 Neurosurg 1983;59:751-61.

8 Brooks D N, Aughton M E, Bond M R, Jones P, Rizvi S. Cognitive sequelae in relationship to early indices of the severity of brain damage after severe blunt head injury. f Neurol Neurosurg Psychiatry 1980;43:529-34.
9 Gronwall D, Wrightson P. Duration of post-traumatic amnesia after mild head injury. $\mathcal{F}$ Clin Neuropsychol 1980;2:51-60.

10 Schacter D L, Crovitz H F. Memory function after closed head injury: a review of the quantitative research. Cortex 1977;13:150-176.

11 Wilson JTL, Hadley DM, Wiedmann KD, Teasdale GM. Intercorrelation of lesions detected by magnetic resonance imaging after closed head injury. Brain Injury 1992;6:391.

12 Hadley D M, Teasdale G M, Jenkins A, et al. Magnetic resonance imaging in acute head injury. Clinical resonance imaging in

13 Levin H S, O'Donnell iv M, Grossman R G. The Galveston Orientation and Amnesia Test: A practical scale to assess cognition after head injury. I Nerv Ment Dis 1979;167:675-684

14 Shores A, Kraiuhin C, Zurynski Y, et al. Neuropsychological assessment and brain imaging technologies in the evaluation of the sequelae of blunt head injury. Aust New Zealand 7 Psychiatry 1990;24:133-8.

15 Annegers JF, Grabow JD, Kurland LT, Laws ER. The incidence, causes, and secular trends of head trauma in Olmstead County, Minnesota, 1935-1974. Neurology, 1980;30:912.

16 Jenkins A, Teasdale G, Hadley M D M, Macpherson P, Rowan J O. Brain lesions detected by magnetic resonance imaging in mild and severe head injuries. Lancet nance imaging

17 Ommaya A K, Gennarelli T A. Cerebral concussion and traumatic unconsciousness: Correlation of experimental and clinical observations on blunt head injuries. Brain 1974;97:633-54.

18 Mayes AR. Human organic memory disorders. Cambridge: Cambridge University Press, 1988. 